\title{
Effect of bone marrow mesenchymal stem cells on experimental pulmonary arterial hypertension
}

\author{
ZHAO-HUA ZHANG ${ }^{1 *}$, YAN LU $^{2 *}, \mathrm{YUN}^{*} \mathrm{LUAN}^{3}$ and JING-JIE ZHAO \\ ${ }^{1}$ Department of Pediatrics, The Second Hospital of Shandong University, Jinan; ${ }^{2}$ Department of Pathology, \\ Hospital of Beijing University of Aeronautics and Astronautics, Beijing; ${ }^{3}$ Central Research Laboratory; \\ ${ }^{4}$ Clinical Molecular Biology Laboratory, The Second Hospital of Shandong University, Jinan, P.R. China
}

Received January 29, 2012; Accepted August 22, 2012

DOI: $10.3892 /$ etm.2012.691

\begin{abstract}
The aim of the present study was to investigate the effect of bone marrow mesenchymal stem cell (BMSC) transplantation on lung and heart damage in a rat model of monocrotaline (MCT)-induced pulmonary arterial hypertension $(\mathrm{PAH})$. The animals were randomly divided into 3 groups: control, PAH and BMSC implantation groups. Structural changes in the pulmonary vascular wall, such as the pulmonary artery lumen area (VA) and vascular area (TAA) were measured by hematoxylin and eosin (H\&E) staining, and the hemodynamics were detected by echocardiography. Two weeks post-operation, our results demonstrated that sublingual vein injection of BMSCs significantly attenuated the pulmonary vascular structural and hemodynamic changes caused by pulmonary arterial hypertension. The mechanism may be executed via paracrine effects.
\end{abstract}

\section{Introduction}

Pulmonary arterial hypertension (PAH) is a progressive disorder characterized by abnormally high blood pressure in the pulmonary artery caused by functional and structural alteration to the pulmonary vasculature resulting in an increase in pulmonary vascular resistance (1). Numerous therapies have been proven useful in decreasing pulmonary arterial pressure, but an effective therapy for the long-term outcome in this disorder is lacking (2-4). Monocrotaline (MCT), a pyrrolizidine alkaloid extracted from the seeds of Crotalaria spectabilis, used in the model of MCT-induced pulmonary hypertension is known to represent similar pathology to that of primary PAH (5).

Correspondence to: Professor Jing-Jie Zhao, Clinical Molecular Biology Laboratory, The Second Hospital of Shandong University, No. 247 Beiyuan Dajie, Jinan 250033, P.R. China

E-mail: jingjiezhao@yahoo.cn

*Contributed equally

Key words: bone marrow mesenchymal stem cells, pulmonary arterial hypertension, pulmonary vascular wall
Bone marrow mesenchymal cells (BMSCs) are multipotent progenitor cells derived from fetal bone marrow, which have the ability to differentiate into bone, cartilage, muscle, bone marrow stroma, endothelial cells, vascular smooth muscle cells $(6,7)$ and other connective tissues. Studies also suggest that BMSCs secrete a variety of growth factors, such as vascular endothelial growth factor (VEGF) $(8,9)$. Recently, BMSC transplantation has become a potential therapy for PAH.

\section{Materials and methods}

Animals. A total of 30 healthy adult Sprague-Dawley (SD) rats weighing 200-250 g were purchased from The Animal Experimental Center of Shandong University, China. The study protocol was reviewed and approved by the Institutional Animal Care and Use Committee, The Second Hospital of Shandong University, and the experiments were conducted according to the Guidelines of the American Physiological Society.

Isolation, culture, immunophenotype analysis and labeling of BMSCs. Bone marrow cells were isolated by flushing the cavity of femurs and tibias, and transferred to a tissue culture dish $90 \mathrm{~mm}$ in diameter $(10,11)$. To separate BMSCs and other cells, the Ficoll (1.077) density gradient centrifugation method was used. The white layer composed of mononuclear cells from the upper layer and interface was carefully collected and washed three times. Flow cytometric immunophenotyping was performed using methods described previously $(12,13)$. Briefly, $5 \times 10^{5}$ cells were suspended with trypsin and were washed twice in phosphate-buffered saline (PBS). Cells were stained with antibodies against CD44, CD29, CD34 and CD90 (BD Biosciences, Franklin Lakes, NJ, USA) for 30 min at $4^{\circ} \mathrm{C}$. The percentage of positive cells was determined by fluorescent activated cell sorting (FACS) analysis. Prior to implantation, the cells were labeled with the cross-linkable membrane dye CM-DiI (2 $\mu \mathrm{g} / \mathrm{ml}$, Invitrogen Corp., Carlsbad, CA, USA) (14). The male SD rats were randomly assigned to 3 groups $(n=10$ in each group) as follows: control group, animals that received a sublingual vein injection of $0.9 \%$ saline instead of BMSCs; PAH group, animals that received a subcutaneous injection of $50 \mathrm{mg} / \mathrm{kg}$ MCT; BMSC group, animals that received $5 \times 10^{5}$ labeled BMSC implantation one week after MCT injection. 


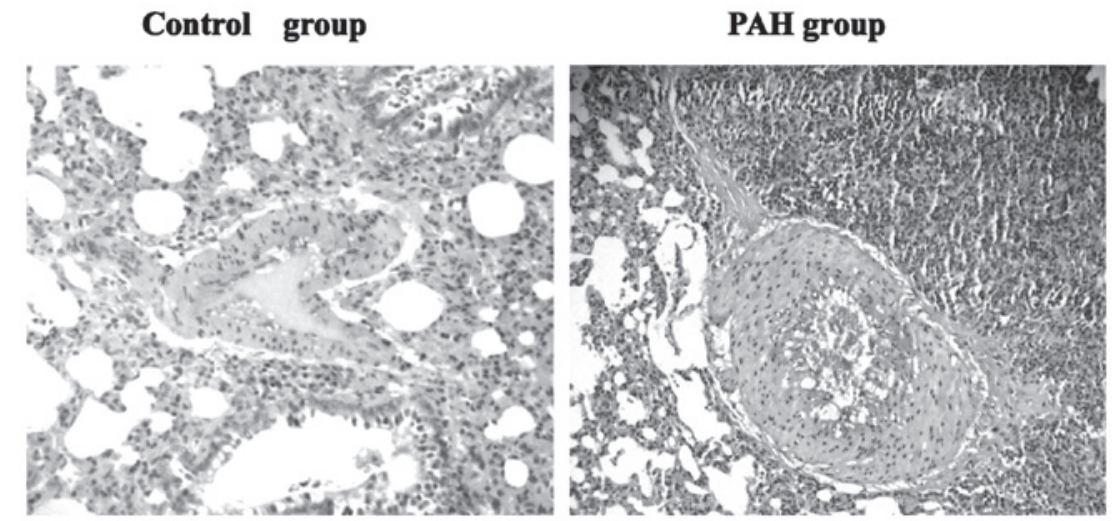

Figure 1. Change in the pulmonary artery wall one week after injection of MCT as determined by H\&E staining (x100 magnification). MCT, monocrotaline; $\mathrm{H} \& \mathrm{E}$, hematoxylin and eosin.

BMSC transplantation. One week after MCT injection, the rats were anesthetized by subcutaneous injection of pentobarbital, $5 \times 10^{5} \mathrm{CM}-\mathrm{DiI}$ labeled BMSCs were resuspended in $100 \mu 1$ saline and administered via the sublingual vein.

Establishment and evaluation of the pulmonary arterial hypertension model. MCT was prepared as described (2) and was dissolved in $1 \mathrm{~N} \mathrm{HCl}$, neutralized to $\mathrm{pH} 7.4$ with $0.5 \mathrm{~N}$ $\mathrm{NaOH}$, and diluted with saline before injection. Male SD rats $(n=10)$ were randomly assigned to 2 groups as follows: PH group, animals that received a subcutaneous injection of $50 \mathrm{mg} / \mathrm{kg} \mathrm{MCT}$; control group, animals that received a subcutaneous injection of $0.9 \%$ saline instead of MCT. One week after MCT injection, the rats were anesthetized and fitted with a 3F-Millar Mikro-Tip catheter via the right jugular vein into the right ventricle to obtain base line measurements of hemodynamics, including right ventricular systolic pressure (RVSP), mean right ventricular pressure (MRVP) and mean pulmonary arterial pressure (MPAP). The rats were sacrificed after hemodynamic measurements, and the lung and heart were quickly harvested and fixed in situ via the trachea cannula with buffered $4 \%$ formaldehyde, and embedded in paraffin. The sections were cut into 4-5 $\mu \mathrm{m}$ slices and were stained with streptavidin peroxidase and hematoxylin and eosin (H\&E). The structural changes in the pulmonary vascular wall were observed by immunofluorescence microscopy and wall thickness (MT), blood vessel diameter (ED), pulmonary artery lumen area (VA) and vascular area (TAA) were measured to calculate MT\% (=MT/ED) and VA\% (=VA/TAA). The ratio of right ventricular weight to body weight (RV/BW) was calculated to measure right ventricular hypertrophy. Results of the hemodynamic parameters, right ventricular hypertrophy, and pulmonary arterial pathological changes were used to evaluate whether the model of pulmonary arterial hypertension was successfully established.

Immunological and immunohistochemical analysis. Two weeks after BMSC transplantation the rats were anesthetized and the lungs were inflated with OCT compound and quickly frozen in liquid nitrogen and stored at $-80^{\circ} \mathrm{C}$. Sections were cut into $4 \mu \mathrm{m}$ slices and fixed in acetone for $10 \mathrm{~min}$ at $-20^{\circ} \mathrm{C}$. The survival of BMSCs was demonstrated by observing the presence of DiI-labeled cells. Immunofluorescence was then
Table I. Effect of MCT on hemodynamics and right ventricular weight one week after administration.

\begin{tabular}{lcc}
\hline Variable & Control & PAH \\
\hline RVSP $(\mathrm{mmHg})$ & $34.38 \pm 1.86$ & $50.33 \pm 1.39^{\mathrm{b}}$ \\
MPAP $(\mathrm{mmHg})$ & $20.18 \pm 2.19$ & $40.28 \pm 3.27^{\mathrm{b}}$ \\
MRVP $(\mathrm{mmHg})$ & $19.43 \pm 3.17$ & $42.64 \pm 4.25^{\mathrm{b}}$ \\
RV/BW & $0.49 \pm 0.03$ & $0.57 \pm 0.06^{\mathrm{b}}$ \\
\hline
\end{tabular}

MCT, monocrotaline; PAH, pulmonary arterial hypertension; RVSP, right ventricular systolic pressure; MPAP, mean pulmonary arterial pressure; MRVP, mean right ventricular pressure; RV/BW, ratio of right ventricular to body weight. ${ }^{\mathrm{a}} \mathrm{P}<0.05,{ }^{\mathrm{b}} \mathrm{P}<0.01,{ }^{\mathrm{c}} \mathrm{P}<0.001$ vs. control.

performed with goat anti-mouse monoclonal surfactant associated protein C (SP-C, 1:100) IgG antibody, rabbit anti-human von Willebrand Factor (vWF, 1:100) antibody and VEGF (1:100) antibody. FITC-conjugated antiserum was used as a secondary antibody.

\section{Results}

The analysis of MCT-induced hemodynamics and $R V$ weight. One week after MCT injection, RVSP, MRVP and MPAP were significantly elevated in the PAH group compared with the control $(\mathrm{P}<0.05)$, and the ratio of RV/BW was significantly higher in the PAH group compared with the control group $(0.49 \pm 0.03$ vs. $0.57 \pm 0.06, P<0.05)$. These results indicated that MCT led to severe right ventricular hypertrophy and the PAH model was successfully established (Table I).

Analysis of MCT-induced structural changes in the pulmonary artery wall. H\&E staining demonstrated significant intima thickening and luminal stenosis in the PAH group compared with the control group. The MT\% of muscular arteries was significantly increased and the VA\% was significantly decreased in the PAH group compared with control group $(\mathrm{P}<0.01$, Table II, Fig. 1). 
Table II. Effect of MCT on the pulmonary artery wall one week after administration.

\begin{tabular}{lcc}
\hline Variable & Control & PAH \\
\hline MT\% & $13.05 \pm 1.33$ & $38.27 \pm 4.55^{\mathrm{b}}$ \\
VA\% & $44.09 \pm 4.17$ & $33.39 \pm 6.63^{\mathrm{b}}$ \\
\hline
\end{tabular}

MCT, monocrotaline; PAH, pulmonary arterial hypertension. MT\% $=\mathrm{MT} / \mathrm{ED}$ and VA\% = VA/TAA $-\mathrm{MT}$, wall thickness; ED, blood vessel diameter; VA, pulmonary artery lumen area; TAA, vascular area. ${ }^{\mathrm{a}} \mathrm{P}<0.05,{ }^{\mathrm{b}} \mathrm{P}<0.01,{ }^{\mathrm{C}} \mathrm{P}<0.001$ vs. control.

Table III. Effect of BMSCs on the hemodynamics and right ventricular weight 2 weeks after implantation.

\begin{tabular}{lccc}
\hline Variable & Control & PAH & BMSCs \\
\hline RVSP (mmHg) & $35.17 \pm 1.86$ & $56.84 \pm 1.54^{\mathrm{b}}$ & $43.83 \pm 2.13^{\mathrm{e}}$ \\
MPAP (mmHg) & $20.36 \pm 2.13$ & $41.37 \pm 2.24^{\mathrm{b}}$ & $26.82 \pm 3.42^{\mathrm{e}}$ \\
MRVP (mmHg) & $19.74 \pm 5.23$ & $41.68 \pm 6.17^{\mathrm{b}}$ & $28.34 \pm 1.98^{\mathrm{e}}$ \\
RV/BW (\%) & $0.472 \pm 0.035$ & $0.613 \pm 0.072^{\mathrm{a}}$ & $0.547 \pm 0.041^{\mathrm{d}}$ \\
\hline
\end{tabular}

BMSCs, bone marrow mesenchymal cells; PAH, pulmonary arterial hypertension; RVSP, right ventricular systolic pressure; MPAP, mean pulmonary arterial pressure; MRVP, mean right ventricular pressure. $\mathrm{RV} / \mathrm{BW}=$ ratio of right ventricular to body weight; ${ }^{\mathrm{a}} \mathrm{P}<0.05,{ }^{\mathrm{b}} \mathrm{P}<0.01$, ${ }^{\mathrm{c}} \mathrm{P}<0.001$ vs. control; ${ }^{\mathrm{d}} \mathrm{P}<0.05,{ }^{\mathrm{e}} \mathrm{P}<0.01$ vs. $\mathrm{PAH}$ group.

Table IV. Effect of BMSCs on the pulmonary artery wall 2 weeks after implantation.

\begin{tabular}{lccc}
\hline Variable & Control & PAH & BMSCs \\
\hline MT\% & $12.08 \pm 1.30$ & $45.21 \pm 4.37^{\mathrm{b}}$ & $20.83 \pm 5.49^{\mathrm{d}}$ \\
VA\% & $42.31 \pm 4.39$ & $20.36 \pm 6.81^{\mathrm{b}}$ & $38.27 \pm 3.48^{\mathrm{d}}$
\end{tabular}

BMSCs, bone marrow mesenchymal cells; PAH, pulmonary arterial hypertension. MT\% = MT/ED and VA\% = VA/TAA - MT, wall thickness; ED, blood vessel diameter; VA, pulmonary artery lumen area; TAA, vascular area. ${ }^{\mathrm{a}} \mathrm{P}<0.05,{ }^{\mathrm{b}} \mathrm{P}<0.01,{ }^{\mathrm{c}} \mathrm{P}<0.001$ vs. control; ${ }^{\mathrm{d}} \mathrm{P}<0.05,{ }^{\mathrm{e}} \mathrm{P}<0.01$ vs. $\mathrm{PAH}$ group.
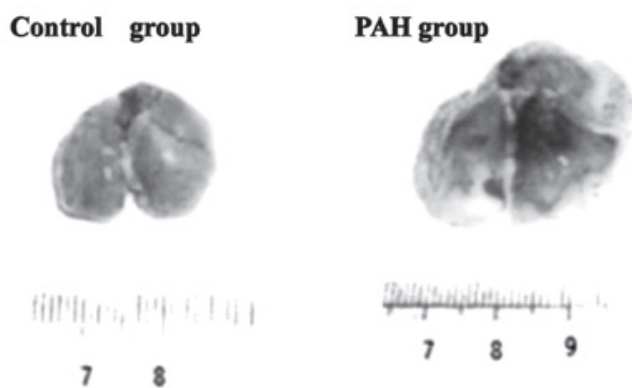

Figure 2. Morphological observation of the lung one week after injection of MCT. MCT, monocrotaline; PAH, pulmonary arterial hypertension.

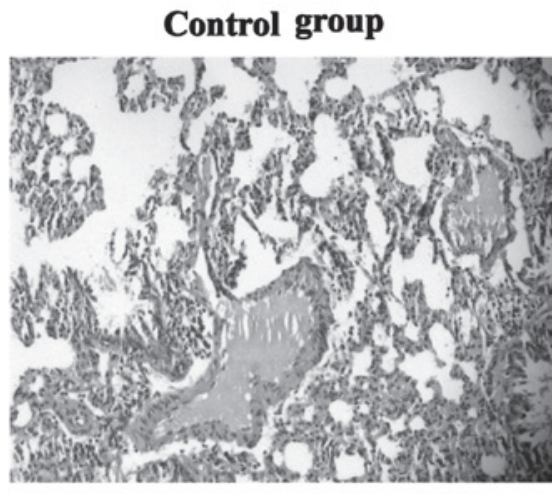

PAH group

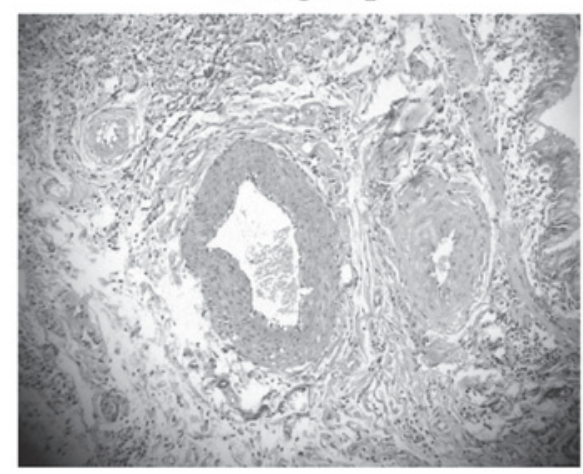

BMSC group

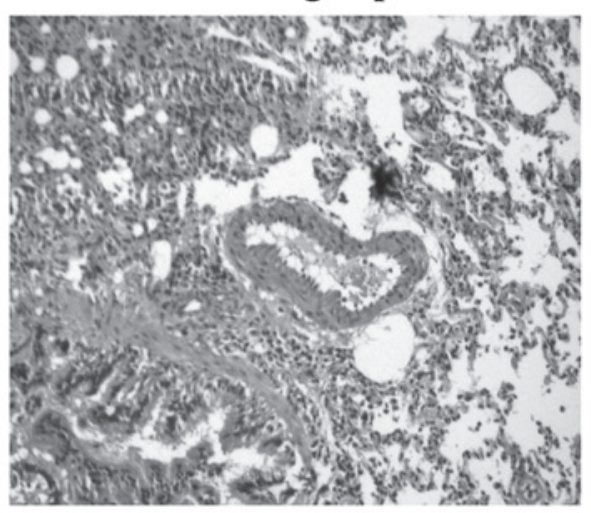

Figure 3. Effect of BMSCs on structural alterations in the pulmonary artery wall as determined by $\mathrm{H} \& \mathrm{E}$ staining 3 weeks after injection (x100 magnification). PAH, pulmonary arterial hypertension; BMSCs, bone marrow mesenchymal cells; H\&E, hematoxylin and eosin.

MCT-induced lung morphological observation. Two weeks after operation, gross observation showed that enlarged volume, purple color, uneven surface, poor elasticity and partial visible blood stasis were present in the PAH group compared with the normal control group (Fig. 2).

Effect of BMSCs on hemodynamics and RV impairment. Two weeks after BMSC administration, RVSP, MRVP and MPAP were significantly lower in the BMSC group compared with the $\mathrm{PAH}$ group, the ratio of RV/BW was significantly lower in the BMSC group compared with the PAH group ( $\mathrm{P}<0.05$, Table III).

Effect of BMSCs on pulmonary artery wall. H\&E staining demonstrated that intima thickening and luminal stenosis were significantly decreased in the BMSC group compared with the 


\section{(VEGF)}

A

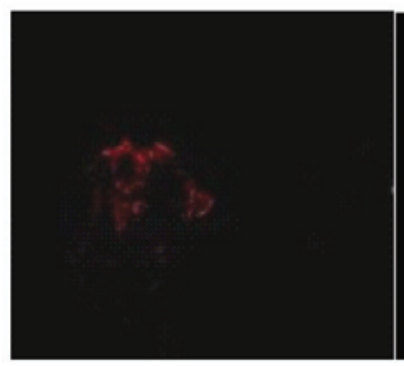

(vWF)
B

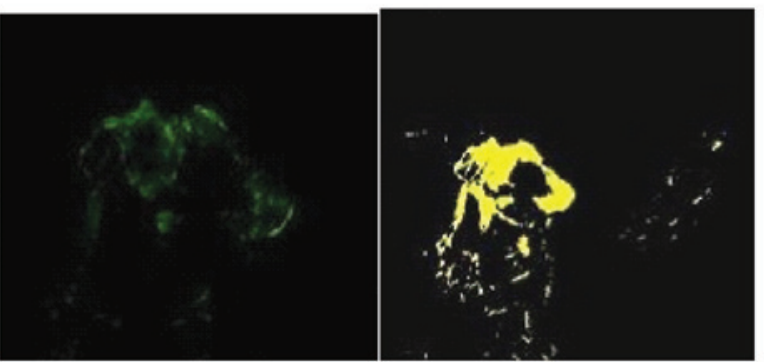

D

$\mathbf{E}$

F

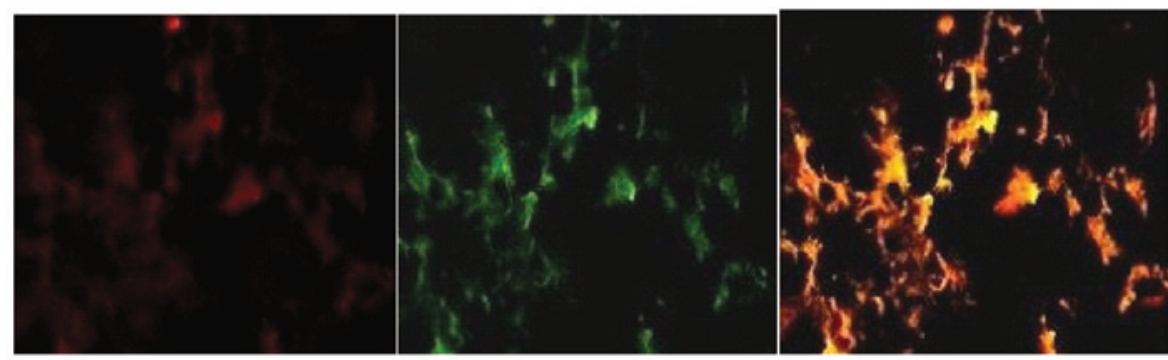

Figure 4. Identification of the transplanted BMSCs. (A and D) Transplanted BMSCs demonstrated red fluorescence, and (B amd E) sections stained with VEGF and vWF produced green fluorescence. (C and F) The merged images show yellow fluorescence (magnification $\mathrm{x} 400)$. There was no evidence of red or green fluorescence in the control or PAH groups. VEGF, vascular endothelial growth factor; vWF, von Willebrand Factor; BMSCs, bone marrow mesenchymal cells.

PAH group. The MT\% and VA\% of muscular arteries was significantly improved in the BMSC group compared with the PAH group ( $\mathrm{P}<0.05$, Table IV, Fig. 3$)$.

Identification of the transplanted BMSCs. In numerous regions, the red fluorescence-positive cells were observed coincident with the green fluorescence spots of VEGF and vWF antibody but not SP-C antibody, which suggested that the intravenous implantation of BMSCs resulted in their differentiation into vascular endothelial cells in vivo although they did not survive as lung cells. There was no evidence of red or green fluorescence in the control and PAH groups (Fig. 4).

\section{Discussion}

PAH is a progressive disorder characterized by the progressive increase in pulmonary arterial pressure and resistance, eventually leading to right heart failure and mortality in patients with refractory disease. Although in the past ten years, the treatment of PAH has achieved apparent progress, the prognosis remains poor. Intravenous administration of drugs (e.g. prostacyclin, endothelin receptor antagonists) or nitric oxide inhalation may temporarily reduce $\mathrm{PAH}$, but these effects are not lasting. In recent years, regeneration and gene therapy has become the research focus worldwide, however, stem cell research is still in its initial stages and so far there is no uniform method to treat PAH. Therefore, for further experimental studies, investigating reasonable and safe methods of treatment for PAH has become urgent.
BMSCs, multipotent progenitor cells derived from fetal bone marrow, could differentiate into distinctive end-stage cell types, including bone, cartilage, muscle, endothelial cells, vascular smooth muscle cells and other connective tissues. Studies have also demonstrated that BMSCs have the pluripotent ability to become endothelial progenitor cells and other cell lineages $(15,16)$. BMSCs are able to secrete a variety of growth factors to promote angiogenesis, such as VEGF.

Transplantation of endothelial progenitor cells (EPCs) into the MCT-injured lung could repair the damage, but the treatment effect is not ideal. The studies on BMSC transplantation for pulmonary hypertension are limited. In our previous research (17), intravenous implantation of BMSCs improved the progression of RV impairment caused by MCT-induced PAH. The aim of this study was to further explore the effect of BMSCs on lung and heart impairment. First, we demonstrated that 2 weeks after sublingual vein administration of BMSCs to PAH rats, the pulmonary arterial pressure was significantly lower in the BMSC group compared with the nontreated PAH group. RVSP, MRVP and MPAP were significantly lower in the BMSC group compared with the $\mathrm{PAH}$ group, the ratio of RV/BW was significantly lower in the BMSC group compared with the PAH group. Notably, in the case of the present study, we found that the structural changes in the pulmonary vascular wall, such as VA and TAA, were significantly improved. Although the underlying mechanisms are complicated and not yet determined, several factors are expected to contribute, including the role of stem 
cell differentiation and para-secretion effects $(3,18-23)$. Our experiments also demonstrated that the red fluorescencepositive cells were observed coincident with the green fluorescence spots of VEGF and vWF antibody but not SP-C antibody in numerous regions. These results suggest that the intravenous implantation of BMSCs results in the ability of these cells to differentiate into vascular endothelial cells in vivo but not lung cells. Therefore, transplantation of BMSCs by homing to the lung and transforming into vascular endothelial cells may create a wide range of collateral circulation, increase the total area of the pulmonary vascular bed, improve pulmonary blood supply and effectively reduce the pulmonary hypertension.

In conclusion, our results showed that intravenous implantation of BMSCs may improve not only the cardiac function and hemodynamics, but also the pulmonary vascular wall damage in PAH caused by MCT. Therefore, this study provides conslusive information for the use of this new method in the treatment of pulmonary arterial hypertension.

\section{Acknowledgements}

This work was supported by a grant from Shandong Province Medical Health Science and Technology Development Programs (2011HZ032) and Independent Innovation Foundation of Shandong University, IIFSDU (grant number 2010TS051).

\section{References}

1. Morrell NW, Adnot S, Archer SL, Dupuis J, Jones PL, MacLean MR, McMurtry IF, Stenmark KR, Thistlethwaite PA, Weissmann N, Yuan JX and Weir EK: Cellular and molecular basis of pulmonary arterial hypertension. J Am Coll Cardiol 54: S20-S31, 2009.

2. Kanki-Horimoto S, Horimoto H, Mieno S, Kishida K, Watanabe F, Furuya $\mathrm{E}$ and Katsumata T: Implantation of mesenchymal stem cells overexpressing endothelial nitric oxide synthases improves right ventricular impairments caused by pulmonary hypertension. Circulation 114: I181-I185, 2006.

3. Baber SR, Deng W, Master RG, Bunnell BA, Taylor BK, Murthy SN, Hyman AL and Kadowitz PJ: Intratracheal mesenchymal stem cell administration attenuates monocrotaline-induced pulmonary hypertension and endothelial dysfunction. Am J Physiol Heart Circ Physiol 292: H1120H1128, 2007.

4. Patel KM, Crisostomo P, Lahm T, Markel T, Herring C, Wang M, Meldrum KK, Lillemoe KD and Meldrum DR: Mesenchymal stem cells attenuate hypoxic pulmonary vasoconstriction by a paracrine mechanism. J Surg Res 143: 281-285, 2007.

5. Miura M, Hirose M, Endoh H, Wakayama Y, Sugai Y, Nakano M, Fukuda K, Shindoh C, Shirato K and Shimokawa H: Acceleration of $\mathrm{Ca}^{2+}$ waves in monocrotaline-induced right ventricular hypertrophy in the rat. Circ J 75: 1343-1349, 2011.

6. Wang $\mathrm{CH}$, Cherng WJ, Yang NI, Kuo LT, Hsu CM, Yeh HI, Lan YJ, Yeh CH and Stanford WL: Late-outgrowth endothelial cells attenuate intimal hyperplasia contributed by mesenchymal stem cells after vascular injury. Arterioscler Thromb Vasc Biol 28: 54-60, 2008.

7. Westerweel PE and Verhaar MC: Directing myogenic mesenchymal stem cell differentiation. Circ Res 103: 560-561, 2008.
8. Gehrke I, Gandhirajan RK, Poll-Wolbeck SJ, Hallek M, Kreuzer KA: Bone marrow stromal cell-derived vascular endothelial growth factor (VEGF) rather than chronic lymphocytic leukemia (CLL) cell-derived VEGF is essential for the apoptotic resistance of cultured CLL cells. Mol Med 17: 619-627, 2011.

9. Crosswhite P, Sun Z: Nitric oxide, oxidative stress and inflammation in pulmonary arterial hypertension. J Hypertens 28: 201-212, 2010

10. Gao J, Dennis JE, Muzic RF, Lundberg M and Caplan AI: The dynamic in vivo distribution of bone marrow-derived mesenchymal stem cells after infusion. Cells Tissues Organs 169: 12-20, 2001.

11. Nagaya N, Fujii T, Iwase T, Ohgushi H, Itoh T, Uematsu M, Yamagishi M, Mori H, Kangawa K and Kitamura S: Intravenous administration of mesenchymal stem cells improves cardiac function in rats with acute myocardial infarction through angiogenesis and myogenesis. Am J Physiol Heart Circ Physiol 287: H2670-H2676, 2004.

12. Choudhary M, Zhang X, Stojkovic P, Hyslop L, Anyfantis G, Herbert M, Murdoch AP, Stojkovic M and Lako M: Putative role of hyaluronan and its related genes, HAS2 and RHAMM, in human early preimplantation embryogenesis and embryonic stem cell characterization. Stem Cells 25: 3045-3057, 2007.

13. Luan Y, Liu XC, Zhang GW, Shi RF, Zhao XB, Zhao CH, Liu TJ, Lü F, Yang Q and He GW: Mid-term effect of stem cells combined with transmyocardial degradable stent on swine model of acute myocardial infarction. Coron Artery Dis 21: 233-243, 2010.

14. Wang Y, Liu XC, Zhang GW, Zhao J, Zhang JM, Shi RF, Huang YZ, Zhao CH, Liu TJ, Song CX, Lü F, Yang Q and He GW: A new transmyocardial degradable stent combined with growth factor, heparin, and stem cells in acute myocardial infarction. Cardiovasc Res 84: 461-469, 2009.

15. Jiang Y, Jahagirdar BN, Reinhardt RL, Schwartz RE, Keene CD, Ortiz-Gonzalez XR, Reyes M, Lenvik T, Lund T, Blackstad M, Du J, Aldrich S, Lisberg A, Low WC, Largaespada DA and Verfaillie CM: Pluripotency of mesenchymal stem cells derived from adult marrow. Nature 418: 41-49, 2002.

16. Oswald J, Boxberger S, Jørgensen B, Feldmann S, Ehninger G, Bornhäuser $\mathrm{M}$ and Werner $\mathrm{C}$ : Mesenchymal stem cells can be differentiated into endothelial cells in vitro. Stem Cells 22: 377-384, 2004

17. Luan Y, Zhang ZH, Wei DE, Zhao JJ, Kong F, Cheng GH and Wang YB: Implantation of mesenchymal stem cells improves right ventricular impairments caused by experimental pulmonary hypertension. Am J Med Sci 343: 402-406, 2012.

18. Frid MG, Brunetti JA, Burke DL, Carpenter TC, Davie NJ, Reeves JT, Roedersheimer MT, van Rooijen N and Stenmark KR: Hypoxia-induced pulmonary vascular remodeling requires recruitment of circulating mesenchymal precursors of a monocyte/macrophage lineage. Am J Pathol 168: 659-669, 2006.

19. Rochefort GY, Vaudin P, Bonnet N, Pages JC, Domenech J, Charbord $\mathrm{P}$ and Eder V: Influence of hypoxia on the domiciliation of mesenchymal stem cells after infusion into rats: possibilities of targeting pulmonary artery remodeling via cells therapies? Respir Res 6: 125, 2005.

20. Tang J, Xie Q, Pan G, Wang J and Wang M: Mesenchymal stem cells participate in angiogenesis and improve heart function in rat model of myocardial ischemia with reperfusion. Eur J Cardiothorac Surg 30: 353-361, 2006.

21. Jiang S, Kh Haider H, Ahmed RP, Idris NM, Salim A, Ashraf M: Transcriptional profiling of young and old mesenchymal stem cells in response to oxygen deprivation and reparability of the infarcted myocardium. J Mol Cell Cardiol 44: 582-596, 2008.

22. Al-Khaldi A, Al-Sabti H, Galipeau J and Lachapelle K: Therapeutic angiogenesis using autologous bone marrow stromal cells: improved blood flow in a chronic limb ischemia model. Ann Thorac Surg 75: 204-209, 2003.

23. Guo Y, He J, Wu J, Yang L, Dai S, Tan X and Liang L: Locally overexpressing hepatocyte growth factor prevents post-ischemic heart failure by inhibition of apoptosis via calcineurin-mediated pathway and angiogenesis. Arch Med Res 39: 179-188, 2008. 